\title{
ANÁLISIS DE LA MOVILIDAD EN ZARAGOZA Y SU INFLUENCIA EN LA CONSTRUCCIÓN DE LOS CINTURONES DE CIRCUNVALACIÓN
}

\author{
José GARRIDO PALACIOS \\ Departamento de Geografía y Ordenación del Territorio \\ Universidad de Zaragoza
}

\begin{abstract}
Resumen: En este trabajo se analiza la movilidad de Zaragoza y sus aspectos más relevantes: la motorización, reparto modal, distribución horaria y los motivos de los viajes, así como los flujos de tráfico en los cinturones previstos.
\end{abstract}

Palabras clave: Movilidad, motorización, modos, motivos de los viajes, distribución horaria y flujos de tráfico.

Abstract: This work analyses the mobility in the town of Zaragoza and the more important aspects: the motorization, modes of travel, distribution of time and the trip purpose, such as the flow of traffic in the futuring road network.

Key words: Mobility, motorization, modes of travel, trip purpose, distribution of time and flow of traffic.

\section{INTRODUCCIÓN}

Uno de los elementos básicos del funcionamiento de la ciudad desde el más puro estructuralismo es sin duda su sistema de transporte, pues por su propia naturaleza es el que traba las diferentes unidades espaciales y las actividades que se desarrollan en ese ámbito. Ahora bien, el sistema de transporte hay que entenderlo como un conjunto de elementos interrelacionados (POTRYKOWSKI y TAYLOR, 1984) donde 1 a movilidad constituye uno de sus componentes principales y cuyo estudio no se limita sólo a estudiar los desplazamientos, sino al conocimiento de sus causas y consecuencias.

El concepto de movilidad denota cierta ambigüiedad, equiparado frecuentemente a la "demanda" o al "tráfico" (ESCALONA, 1989); sin embargo, la movilidad puede definirse como "un proceso integrado en el que intervienen múltiples actores con múltiples motivos (consumo, ocio, etc.) que juegan un papel común: movilidad 
residencial, de trabajo, de recreo" (NIJKAMP y REICHMAN, 1987), o bien como "una variable cuantitativa que mide simplemente la cantidad de desplazamiento que las personas o las mercancías efectúan en un determinado sistema o ámbito socioeconómico" (ESTEVAN, 1996, 43). Ello implica un abanico muy amplio de posibilidades, por lo que parece obligado acotar el campo de análisis y centrarse en unos aspectos muy concretos. Así pues, nos vamos a limitar al estudio de la movilidad de la población en el municipio de Zaragoza y a deducir, en función de los desplazamientos de vehículos, los flujos y repercusiones que conlleva la construcción del III y IV cinturón de la ciudad ${ }^{1}$, en virtud de que estas infraestructuras constituyen uno de los puntos débiles de la ciudad y que el propio desarrollo urbano y metropolitano demanda (EBRÓPOLIS, 1996) ${ }^{2}$.

El estudio de la movilidad de la población engarza con la óptica desagregada, que aporta abundante información sobre los desplazamientos de los individuos a lo largo del día. Esta unidad de análisis permitirá, por tanto, conocer los motivos de los desplazamientos, modos de transporte utilizados, destinos, horarios, etc. (ESCALONA, 1989).

Los aspectos más relevantes de la movilidad considerados en este trabajo son: análisis de la motorización en función de la población, el reparto modal de los desplazamientos, la distribución horaria y los motivos de la movilidad, así como los flujos en los cinturones proyectados y su influencia en los desplazamientos urbanos. No se analiza el ferrocarril porque su incidencia en la movilidad urbana es prácticamente nula, al par que su función está ligada a los desplazamientos de carácter regional y nacional. Por razones similares no se contemplan los viajes por vía aérea, cuya función está vinculada con la interconectividad nacional e internacional.

Para el trabajo hemos utilizado dos fuentes de estudio principales: la Encuesta de Origen y Destino (O-D) realizada por el Ayuntamiento de Zaragoza en 1993 y los valores de Intensidad Media Diaria (IMD) en la red viaria de los accesos a la ciudad. La primera constituye la fuente básica de los estudios sobre desplazamientos de la población en un área dada, así como de la planificación de la demanda de transporte (SEGUÍ, 1991). Los valores de la IMD permiten conocer el tráfico en las principales vías de acceso y de circunvalación de la ciudad.

\footnotetext{
1 Por motivos de extensión, no se quiere entrar en ęl papel que juegan las infraestructuras en la conformación del Area Metropolitana de Zaragoza, donde las Administraciones Públicas disponen de un excelente arma de cara a implantar un modelo racional de crecimiento urbano.

2 EBROPOLIS (1996): Zaragoza hoy: Síntesis del diagnóstico estratégico, Asociación para el Desarrollo Estratégico de Zaragoza y su área de influencia, 139 pág., Zaragoza.
} 


\section{MOVILIDAD GLOBAL Y MOTORIZACIÓN}

La movilidad global de Zaragoza en 1993, medida en desplazamientos por habitante y día, fue de 2.4 , cifra que se considera muy baja y que está ligada a 1 elevado potencial de población mayor de 65 años (el 14.3\% del total en 1991), lo que obviamente condiciona el número de desplazamientos. No obstante, el crecimiento reciente de la movilidad ha sido notable, pues se ha incrementado 0.6 desde 1986, 10 cual puede ser una consecuencia directa del incremento de las rentas de la población, mejora de las infraestructuras y la incorporación de nuevos hábitos sociales.

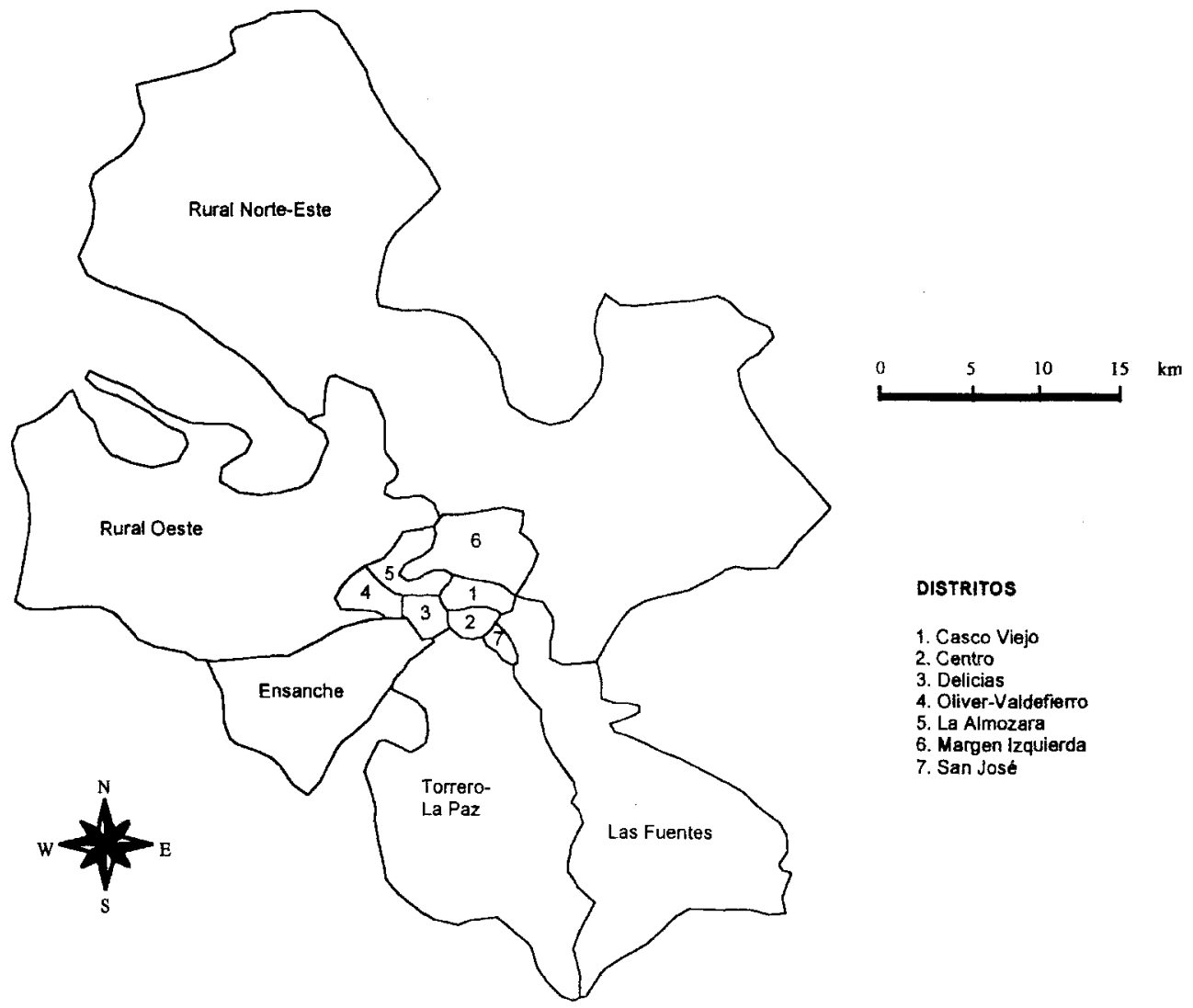

Mapa 1.- Distritos del municipio de Zaragoza, 1998. 
Por su parte, la tasa de motorización en el municipio de Zaragoza es de 269 turismos por cada 1000 habitantes o, lo que es lo mismo, un turismo por cada 3,7 habitantes. Este valor está muy próximo al promedio de la región aragonesa (276 turismos/1000 habitantes), sobre el que incide de forma singular por residir en la capital en torno a la mitad de la población y disponer de gran parte de los turismos de la región (69.7\% de Aragón), lo que indica el alto grado de polarización demográfica y de motorización del área urbana sobre el conjunto regional. Sin embargo, esos valores están por debajo de la media nacional (305 turismos/1000 habitantes), lo cual parece señalar que en la Comunidad Autónoma de Aragón y por extensión en Zaragoza influyen una serie de factores específicos que determinan esa situación.

No consideramos que la baja tasa de motorización sea consecuencia directa de 1 a renta regional, pues los estudios del Banco Bilbao Vizcaya ${ }^{3}$ sitúan a Aragón por encima de la media nacional; por tanto, los factores que parecen condicionantes de 1 a tasa de referencia son: el elevado índice de envejecimiento ${ }^{4}$, lo que limita la utilización del turismo en los desplazamientos; la amplia captación de viajeros en transporte colectivo, como se verá más adelante; y la propia distribución de la población. En este sentido nos ha parecido oportuno desagregar la unidad de análisis (el municipio de Zaragoza) en 12 distritos y hacer un muestreo de los más representativos. Así, los distritos que ofrecen mayor desigualdad son los del casco urbano central (Casco Viejo y Centro) y la periferia (distritos de los barrios rurales Norte-Este y Oeste). De hecho, en la tabla adjunta se aprecia el fuerte contraste demográfico entre los distritos interiores (el Centro con más de 63.000 hab. y el Casco Viejo con 40.000 hab.) y la periferia (distritos rurales entre 11.000 y 15.000 hab.).

Tabla 1. -Indice de motorización y movilidad de la población.

\begin{tabular}{|lcc|}
\hline \multicolumn{1}{|c}{ ZONAS } & TURISMO/1.000 HAB. & POBLACION (Padrón 1991) \\
\hline Casco Viejo & 219 & 40755 \\
Centro & 282 & 63490 \\
Rural Norte-Este & 339 & 15755 \\
Rural Oeste & 320 & 11375 \\
Media Zaragoza & 269 & 600775 \\
\hline
\end{tabular}

Fuente: Ayuntamiento de Zaragoza (1993).

\footnotetext{
${ }^{3}$ Diversos estudios sobre la Renta Familiar Disponible per cápita de Aragón así lo avalan (Banco Bilbao Vizcaya, Renta nacional de España).

${ }^{4}$ En el municipio de Zaragoza esa tasa alcanzó el 0.77 en 1991, lo que implica una población muy envejecida. Para su cálculo se han tenido en cuenta los habitantes mayores de 60 años y los menores de 20 años.
} 
De igual modo, la tabla refleja la alta tasa de motorización de los barrios rurales (más de 320 turismos / 1000 hab.) en detrimento principalmente del Casco Viejo, lo que coincide con la necesidad de desplazamiento de los distritos periféricos por distintos motivos, en especial por el trabajo. Además debe añadirse la residencia de población joven con niveles de renta más elevados que la media municipal, y sobre todo muy superior a la población residente en el Casco Viejo. Este aspecto puede estar también en relación con la alta tasa de motorización del Centro (282 turismos/1000 hab.), pues corresponde a un distrito donde el precio del suelo es muy elevado y donde se concentran gran parte de los servicios ${ }^{5}$ de Zaragoza.

\section{LOS MODOS, MOTIVOS Y FRECUENCIA HORARIA DE LA MOVILIDAD}

Entre los modos de transporte hay que distinguir los motorizados y los no motorizados. Los primeros pueden ser públicos (autobús, metro, etc.), semipúblicos (taxis) y privados (automóvil y motocicleta en calidad de conductor o de pasajero). Los transportes no motorizados comprenden los desplazamientos a pie o en bicicleta.

Los desplazamientos ${ }^{6}$ a pie son los predominantes en el interior de Zaragoza (el $42 \%$ ), lo que supone un elevado porcentaje en comparación a otras ciudades españolas (pues están en torno al 38\%). Los viajes en autobús ${ }^{7}$ captan el $30 \%$ de los desplazamientos diarios, cifra que también supera a otras urbes (SEGUÍ, 1991). A este respecto ha contribuido sustancialmente el precio del billete (subvencionado por el Ayuntamiento) y la calidad del transporte.

En cualquier caso, los transportes colectivos deben ser utilizados como elemento básico de planificación territorial y representativos de la calidad de vida de los ciudadanos, por lo que en este sentido la política aplicada en la capital aragonesa puede ser más o menos acertada pero sin duda ha reducido la movilidad en vehículo privado, con las subyacentes ventajas de descongestión, ahorro de energía y coste medioambiental.

\footnotetext{
${ }^{5}$ En este sentido cabe recordar la estructura interna de las ciudades manifestada por Precedo (1988), de modo que en la unidad central es donde se localiza la doble función comercial y residencial.

${ }^{6}$ En todos los casos solamente se han tenido en cuenta los movimientos durante los días laborables por ser los más representativos. De cualquier forma, las variaciones de los sábados y domingos respecto a los anteriores no son muy significativas.

${ }^{7}$ En 1991 se alcanzó la cifra mágica de los 100 millones de viajeros anuales, si bien ha descendido ligeramente en 1994 (99.442.544 viajeros). A modo de comparación, en Sevilla viajan 79 millones, en Valencia 105 millones y en Barcelona el autobús capta 173 millones de viajeros.
} 
Tabla 2. -Distribución modal.

\begin{tabular}{|lcc|}
\hline \multicolumn{1}{|c}{ MODOS } & EXTERIOR DE ZAR. (\%) & INTERIOR DE ZARA. (\%) \\
\hline Automóvil privado & 68 & 25 \\
Autobús & 2 & 30 \\
Pie & 1 & 42 \\
Otros & 29 & 3 \\
\hline TOTAL & 100 & 100 \\
\hline
\end{tabular}

Fuente: Ayuntamiento de Zaragoza.

El porcentaje de los desplazamientos en automóvil es del $25 \%$ en el interior de Zaragoza, cifra similar a la media de las ciudades españolas $(26,5 \%)$, mientras que ese umbral se eleva sustancialmente $(68 \%)$ cuando se refiere a las relaciones con el exterior del municipio. Este hecho es obvio por la reducción de los viajes a pie de larga duración, aunque el bajo nivel de utilización del autobús es otro dato de singular importancia, ya que implica su necesaria atención en aras de obtener un servicio público interurbano de calidad. En este modo de transporte cabe añadir el notable valor porcentual de "otros" (29\%) definidos por los desplazamientos en taxis y los gestionados por las empresas.

Los motivos de los desplazamientos están ligados a la atracción de las áreas urbanas con relación a la distribución de la población activa y las actividades que desarrollan. Así, el $44 \%$ de los viajes corresponden al regreso al hogar, el $22 \%$ a 1 a movilidad obligada (trabajo, visitas al trabajo y estudio), el.19\% a la movilidad no obligada (compras, ocio y visitas) y el $15 \%$ a la movilidad mixta (comida/cena y asuntos personales). En cualquier caso, los viajes por motivos de trabajo constituyen el grueso de los desplazamientos de la movilidad obligada (el $57 \%$ de este agrupamiento) en función de la relativa concentración de la actividad y la dispersión espacial de la residencia. Ahora bien, una de las características más importantes de la movilidad es su dimensión temporal. Así, mediante tablas cruzadas se relacionan los viajes por modos y motivos con los períodos horarios en que se ha fraccionado el día completo.

En las Figuras 1 y 2 se observa el predominio de los desplazamientos a pie a lo largo de la jornada excepto durante la noche, donde el automóvil privado y los taxis suplen a los primeros. 


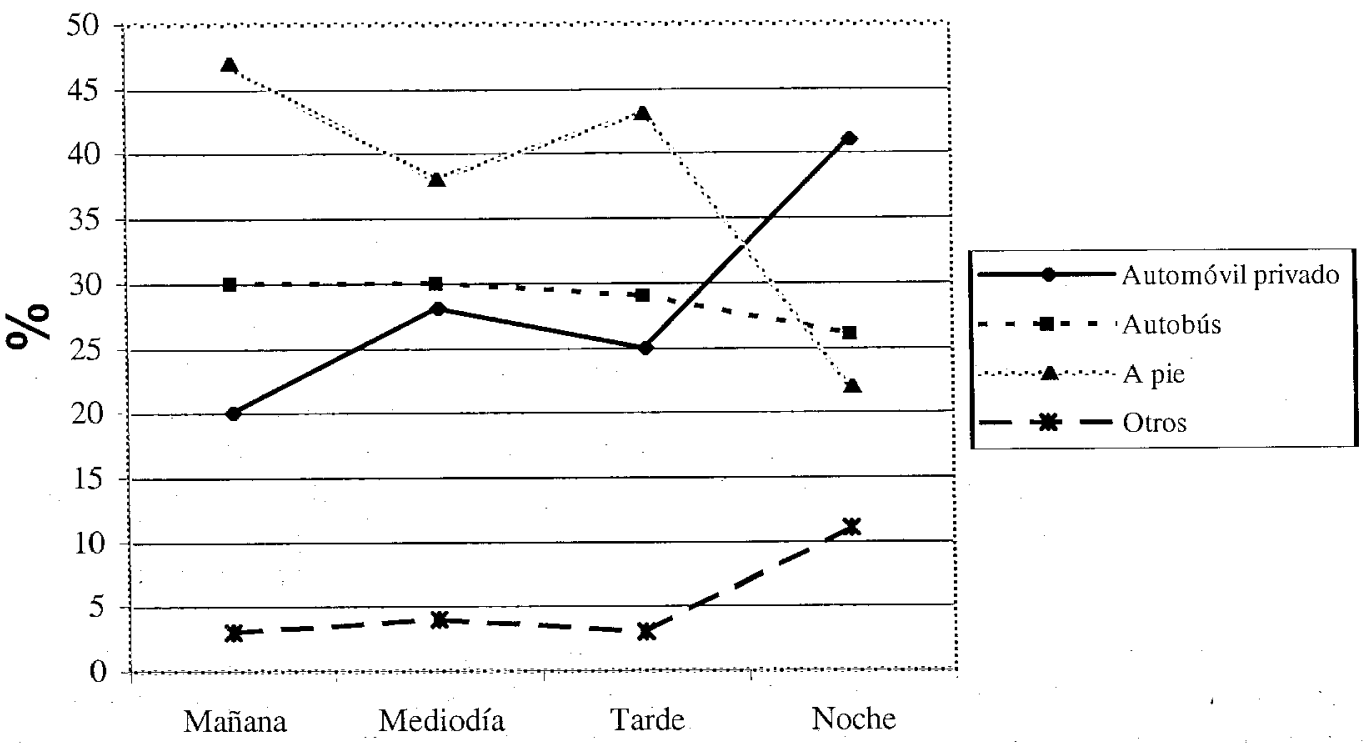

Figura 1.- Reparto modal y frecuencia horaria.

Fuente: Ayuntamiento de Zaragoza (1993).

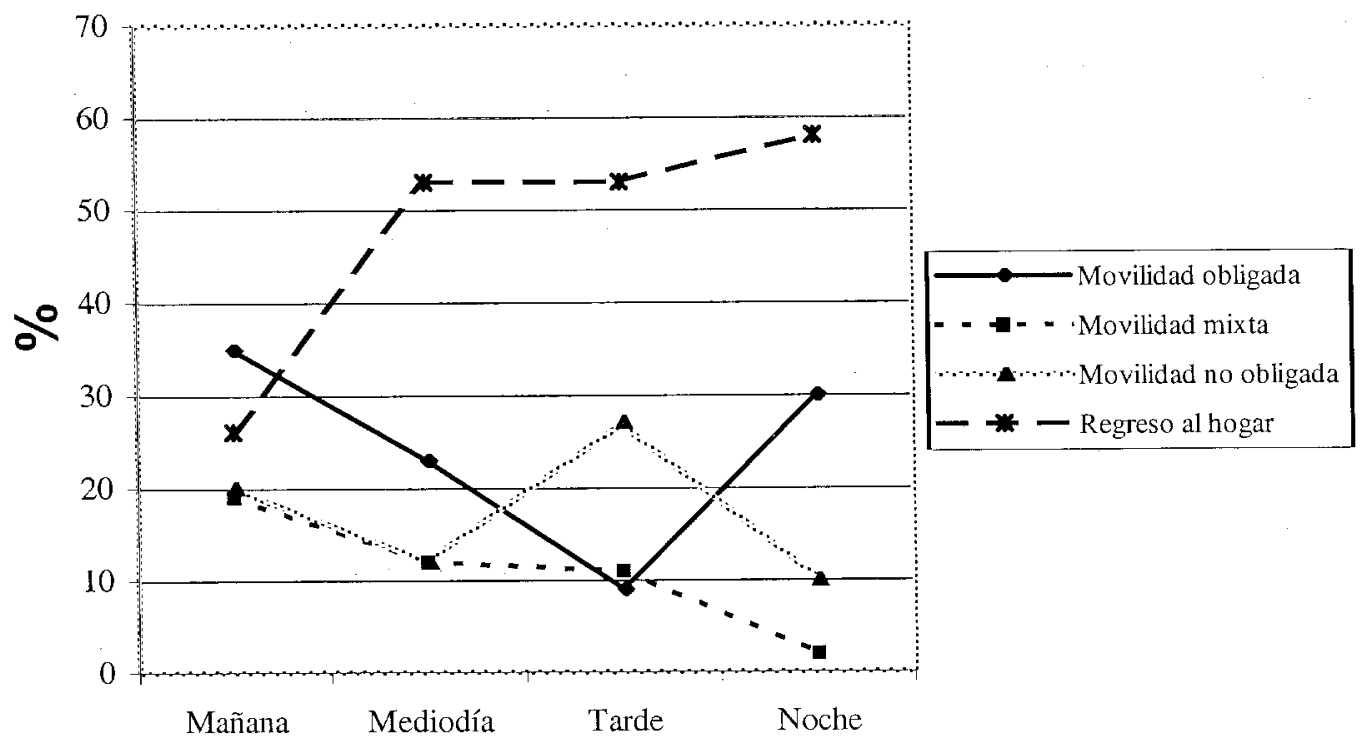

Figura 2.- Motivos de la movilidad y frecuencia horaria. Fuente: Ayuntamiento de Zaragoza (1993). 
Por otro lado, la utilización del autobús se mantiene constante durante el tramo diurno, mientras que por la noche desciende ligeramente en beneficio de los mismos modos apuntados anteriormente. En consecuencia, las diferencias más notables son las reflejadas durante la noche, de forma que los desplazamientos a pie son sustituidos por el automóvil particular y el taxi.

Sin embargo, la diferente movilidad urbana está más condicionada por razones sociales y económicas que la variación del día o la noche. Los desplazamientos regulares corresponden al trabajo y estudios, mientras que las compras y el ocio pierden normalmente esa sincronización.

El desplazamiento por motivos de regreso al hogar es el de mayor porcentaje, como ya se ha apuntado, siendo constante del mediodía en adelante. Esto parece lógico en función de la finalización de la jornada laboral, estudios o el regreso a casa para el descanso nocturno.

En la tabla 3 se refleja asimismo la movilidad no obligada (compras y ocio) durante el período de la tarde, lo que coincide con los hábitos de paseo, compras, visitas a particulares, etc. una vez terminada la jornada de trabajo ${ }^{8}$. Por ello, la importancia de los desplazamientos durante ese tramo horario es muy significativa en determinadas zonas, en especial las comerciales (el núcleo central de Zaragoza y los accesos a las grandes superficies).

Hasta aquí hemos visto diversos aspectos de la movilidad urbana de Zaragoza, que, grosso modo, no difiere de otras ciudades y en función de los desplazamientos a pie, en autobús y la tasa de motorización no parece que existan grandes problemas de movilidad. Ahora bien, esto no indica la ausencia de proyectos para mejorar la situación actual y resolver ciertos desajustes de la ciudad.

En este sentido, Zaragoza carece de carretera de circunvalación que evite el tráfico de paso de norte a sur o de oeste a este por la zona meridional de la ciudad, lo que comporta problemas de congestión en el interior, accidentes, ocupación del espacio urbano y otros de tipo medioambiental como el ruido, emisiones contaminantes, etc. Por tanto, parece importante analizar este aspecto como un elemento imprescindible de la planificación.

8 En el mismo sentido se puede señalar que el mayor flujo de tráfico por la encrucijada de la Plaza del Paraíso se produce entre las 7 y las 9 de la tarde, siendo superior por tanto a las consideradas horas punta de entrada o salida del trabajo o del colegio. 

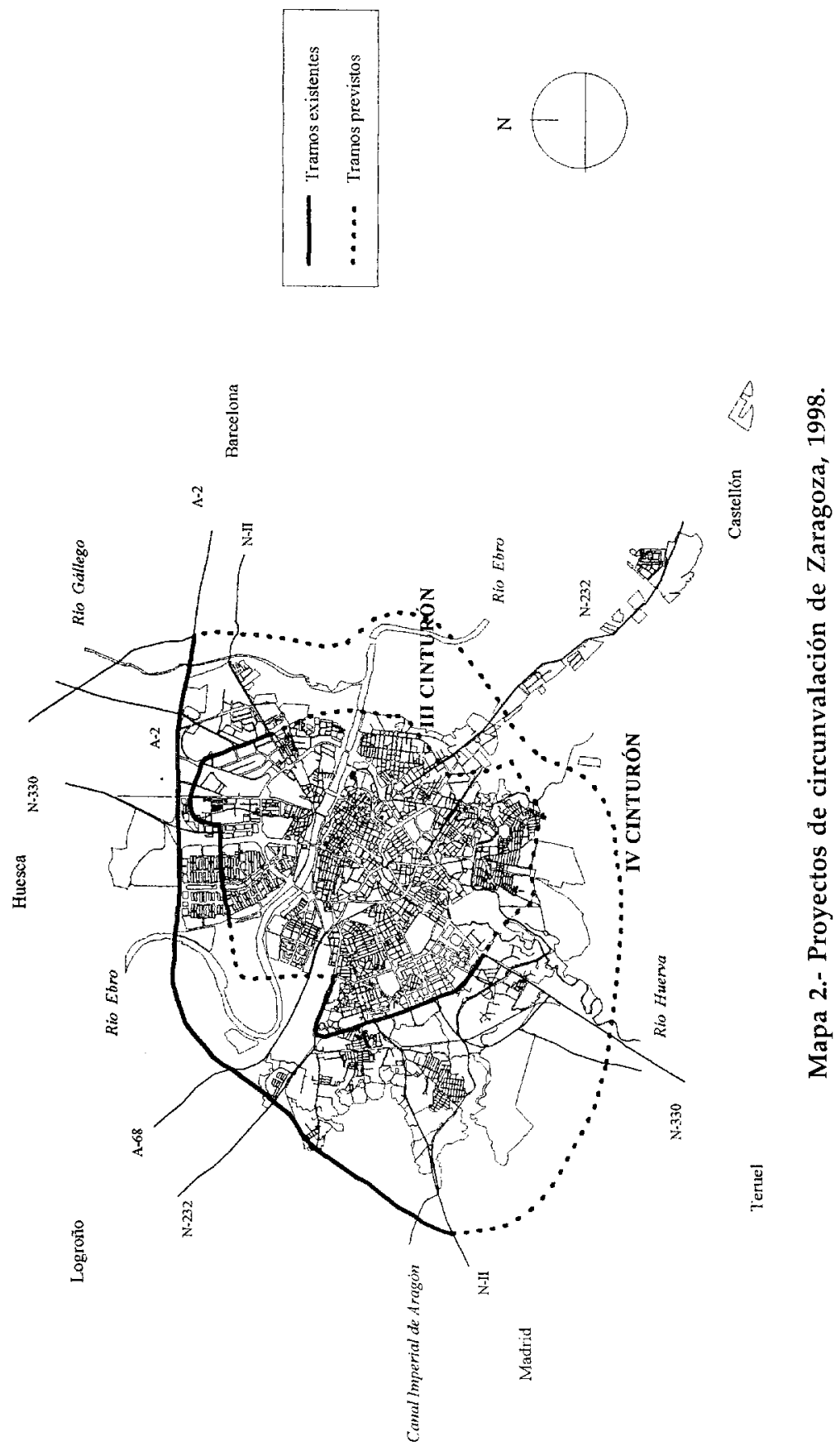


\section{LAS NUEVAS VÍAS DE CIRCUNVALACIÓN DE LA CIUDAD}

Se ha debatido con cierta profusión la posible necesidad de las nuevas vías de circunvalación o Cinturones ${ }^{9}$ III y IV de la ciudad y acerca de sus trazados, pero este trabajo no entra en esa polémica, pues tan sólo pretendemos señalar las variaciones de movilidad que puede experimentar el municipio de Zaragoza en caso de construcción de los mismos (Mapa 2).

Para ello se ha utilizado la fuente señalada de los mapas de tráfico (IMD) del Ayuntamiento de la ciudad y del MOPTMA. Así, en función del tráfico actual vamos a predecir en líneas generales las posibles intensidades de las nuevas vías y su influencia en la movilidad interior de la ciudad.

Pero el tráfico se entiende de diferentes maneras, siendo frecuente su acepción como "problema", cuando en realidad la población es el agente que lo provoca para obtener unos fines determinados. Así pues, RAMOS (1996) ${ }^{10}$ define el tráfico como "el intermediario entre unas causas (concentración, densidad urbana, necesidad de movilidad, hábitos culturales y sociales, etc.) y unos efectos (las disfunciones urbanas: congestión, ruido, contaminación, accidentes, etc.)".

Dicho lo anterior, nos interesa saber sobre qué redes se implementa ese tráfico y qué funciones van a cumplir las nuevas infraestructuras. En primer lugar, el proyecto del III Cinturón, que se extiende desde la Vía Tbérica hasta su conexión con la A-2 a 1 a altura de Mercazaragoza con una longitud de $10.2 \mathrm{~km} .{ }^{11}$., tiene una función eminentemente urbana, con capacidad de absorber gran cantidad de tráfico. Estructura los límites de la ciudad compacta y reequilibra las diferentes partes de la misma, cerrando el anillo incompleto que se corta en la carretera de Valencia y la salida hacia Barcelona.

El IV Cinturón discurre desde la carretera de Madrid (Feria de Muestras), conectando las carreteras de Valencia y Castellón y enlazando con la A-2 en las proximidades de Alfajarín. Su función es metropolitana y de canalización de los flujos

9 El diseño de "cinturón" como elemento vertebrador de la región se basa fundamentalmente en soluciones alejadas del núcleo central, soluciones que tienden a unir de la manera más directa posible la primera corona de núcleos periféricos de importancia. Además se complementarán con otros distribuidores del núcleo central con diseño urbano situados muy próximos al continuo consolidado y con una gran accesibilidad desde dicho continuo.

${ }^{10}$ Según la Conferencia impartida por A. Ramos con el título "Tráfico y transporte en la ciudad de Zaragoza. Una visión global" dentro del ciclo Grandes Vías Urbanas y Construcción de la Ciudad celebrado en Zaragoza durante los meses de enero y febrero de 1996.

11 Si bien la Coordinadora de Venecia-Torrero-La Paz propuso otro trazado alternativo con 1200 metros más de longitud. 
interurbanos. Actúa además como distribuidor de las vías de penetración a la ciudad. Se considera que esta infraestructura es vital para la consolidación del Area Metropolitana de Zaragoza, el eje interurbano Norte-Sur y el desenclavamiento de los corredores de Castellón y Valencia.

Con todo, "los cinturones proyectados pueden evitar que el tráfico de paso colapse las vías internas" y además "transmitirán sus efectos beneficiosos al Area Metropolitana al propiciar la creación planificada de nuevos espacios mejor comunicados fuera del centro congestionado" (CALVO PALACIOS, 1995).

Pero bien, ¿cuál es la variación de movilidad que experimenta la ciudad con la construcción de estas infraestructuras? Para ello hemos calculado el número de viajes motorizados que la población de las zonas afectadas realiza a los distritos por los que discurre el previsto III Cinturón (vid Tabla 3), y esto nos proporciona unos porcentajes respecto al total de los viajes. Siendo estos datos las variaciones de movilidad que experimentaría cada zona con las nuevas vías, oscilando entre el 19.3\% en el Ensanche y el $11.1 \%$ en Las Fuentes, de modo que la media alcanza el $15.92 \%$.

Por otro lado se conocen los flujos de tráfico que acceden al interior de la ciudad; así pues, aplicando los mismos valores porcentuales anteriores a estos flujos ${ }^{12}$ se obtienen los valores posibles de intensidad de tráfico en cada una de las vías radiales afectadas por este Cinturón, y que si en la actualidad se dirigen al centro para conectar con otro distrito, con la nueva construcción utilizarían el Cinturón para la misma finalidad. Con todo, el flujo total asciende a 22.906 vehículos al día.

Tabla 3. - Flujos previstos en el III Cinturón.

\begin{tabular}{|c|c|c|c|c|c|c|}
\hline ZONAS & VIAJES OTRAS ZONAS & VIA PRINCIPAL I & ALVIA & PORCENTAJE & FLUJOS VEHICULOS & FLUJOS PREVISTOS \\
\hline Rural Oeste & 4423 & N-II (a Madrid) & 22952 & 19,2 & 20830 & 3999 \\
\hline Ensanche & 7119 & N-330 (a Valencia) & 36981 & 19,3 & 40130 & 7725 \\
\hline Torrero-La Paz & 7194 & & 58176 & 12,4 & & \\
\hline Las Fuentes & 10112 & N-232 (a Castellón) & 90697 & 11,1 & 20520 & 4820 \\
\hline Rural Norte-Este & 6417 & A-2 (a Barcelona) & 36439 & 17,6 & 36140 & 6360 \\
\hline TOTAL & & & & & & 22906 \\
\hline
\end{tabular}

Fuente: Ayuntamiento de Zaragoza (1993) y Mapa de Tráfico (1993).

El mismo criterio se aplica al IV Cinturón, como se puede observar en la tabla adjunta, de manera que el flujo total se eleva a 29.612 vehículos diarios, siendo mayor al anterior porque lógicamente parte del tráfico no penetra en la ciudad, sino que finaliza su trayecto en la periferia o bien se dispersa por otras vías de menor entidad.

12 Se aplica el mismo criterio sobre las vías principales de acceso a Zaragoza afectadas por el III Cinturón. En cuanto a los flujos que discurren por la autopista A-2 y la carretera nacional N-II en dirección a Barcelona, se han sumado los valores de ambas y sólo se expresa la primera. 
Tabla 4. - Flujos previstos en el IV Cinturón.

\begin{tabular}{|llccc|}
\hline \multicolumn{1}{|c}{ ZONAS } & VIA PRINCIPAL & PORCENTAJE & FLUJOS VEHICULOS & FLUJOS PREVISTOS \\
\hline Rural Oeste & N-II (a Madrid) & 19,2 & 28660 & 5502 \\
Ensanche & N-330 (a Valencia) & 19,3 & 43560 & 8385 \\
Torrero-La Paz & & 12,4 & & \\
Las Fuentes & N-232 (a Castellón) & 11,1 & 27620 & 6490 \\
Rural Norte-Este & A-2 (a Barcelona) & 17,6 & 54328 & 9235 \\
TOTAL & & & & 29612 \\
\hline
\end{tabular}

Fuente: Ayuntamiento de Zaragoza (1993) y Mapa de Tráfico (1993).

No obstante, estas previsiones se refieren al año 1994, por lo que parece necesario conocer cuál sería la intensidad de tráfico, por ejemplo, en el año 2000. En este sentido, la evolución del parque de vehículos desde 1988 a 1994 fue del $21.6 \%$, por lo que en los próximos seis años el incremento puede ser el mismo, y esto produciría la misma variación de movilidad de vehículos. Por consiguiente, la intensidad en el III Cinturón en el año 2000 puede ser de 29.179 vehículos/día y en el IV Cinturón de 36.563 vehículos/día, superando el flujo actual de las carreteras de Madrid (N-II) y Castellón (N-232) a la entrada de Zaragoza.

En todos los casos hemos considerado la existencia de uno de los dos Cinturones precitados, pero qué variación se produce si contemplamos la construcción de ambos. Pensamos que una aproximación sería la partición del flujo total entre las dos vías, lo que representaría en el año 2000 unos 18.000 vehículos/día por cada Cinturón, aunque en función de los resultados anteriores el flujo por el IV Cinturón puede ser algo mayor.

\section{CONCLUSIÓN}

El estudio nos ha permitido conocer la baja movilidad global de Zaragoza, ligada fundamentalmente al envejecimiento de la población. De igual modo, la reducida tasa de motorización depende de la estructura demográfica, su distribución y la amplia captación del transporte en autobús. Este modo de transporte, junto a los desplazamientos a pie, infiere menor utilización del vehículo privado en el interior de la ciudad, lo que propicia la descongestión, menor ocupación del suelo y mayor calidad medioambiental.

Por otra parte, los Cinturones previstos que cierran los anillos exteriores de Zaragoza por su parte meridional y oriental mejoran sustancialmente el tráfico interior y la interconectividad de la ciudad. 
En definitiva, si la movilidad no representa en la actualidad un problema acuciante, si lo puede suponer en el futuro; por tanto, parece adecuado planificar y ejecutar lo necesario en aras de obtener mayor calidad de vida de la población.

\section{BIBLIOGRAFÍA}

AYUNTAMIENTO DE ZARAGOZA (1993): Encuesta Origen-Destino, Ayuntamiento de Zaragoza. Zaragoza.

BBV (1992): Renta nacional de España 1989. Avance de 1990 y 1991, Estudios del Banco Bilbao Vizcaya, 363 p., Bilbao.

CALVO PALACIOS, J.L. (1995): "El Eje de desarrollo del Valle del Ebro y las perspectivas del Eje Norte-Sur de Aragón", III Congreso de Economía Aragonesa, 341-374, Zaragoza.

EBRóPOLIS (1996): Zaragoza hoy: Síntesis del diagnóstico estratégico, Asociación para el Desarrollo Estratégico de Zaragoza y su área de influencia, 139 p., Zaragoza.

ESCALONA ORCAO, A. (1989): "Tendencias actuales de la Geografía del transporte: análisis de la movilidad", Geographicalia, 26, Universidad de Zaragoza, 83-88, Zaragoza.

ESTEVAN, A. y SANZ, A. (1996): Hacia la reconversión ecológica del transporte en España,

MOPTMA (1993): Mapa de tráfico 1993, Dirección General de Carreteras, Madrid.

MINISTERIO DE FOMENTO (1995): Mapa de tráfico 1995, Dirección General de Carreteras, Madrid.

NIJKAMP, P. y REICHMAN, S. (1987): Transportation Planing in a Changing World, European Science Foundation, Strasbourg.

POTRYKOWSKI, M. y TAYLOR, Z. (1984): Geografía del transporte, Ariel, 303, Barcelona.

PRECEDO LEDO, A. (1988): La Red Urbana, Síntesis, 159 p., Madrid.

SEGUI PONS, J.M. y PETRUS BEY, J.M. (1991): Geografía de redes y sistemas de transporte, Síntesis, 231 p., Madrid. 